\title{
THE 3-MODULAR CHARACTERS OF THE RUDVALIS SPORADIC SIMPLE GROUP AND ITS COVERING GROUP
}

\author{
GERHARD HISS
}

\begin{abstract}
The decomposition numbers of the Rudvalis sporadic simple group and of its double covering group modulo 3 are completely determined. The results were obtained using the computer algebra system MOC developed by Jansen, Lux, Parker, and the author, but the proofs are given in conventional form and can be verified by hand with the ordinary character table.
\end{abstract}

\section{Results}

In the present paper we determine the 3-modular decomposition matrices for the sporadic group $G=2 \mathrm{Ru}$, the double cover of the Rudvalis group. We begin by presenting the results. For each nontrivial block in turn we display the table of decomposition numbers followed by a table giving the degrees of the irreducible Brauer characters in the block as well as the factorization of these degrees into prime factors. Projective indecomposable characters are denoted by upper case $\Phi$ 's and the corresponding irreducible Brauer characters by lower case $\phi$ 's.

1.1. Block 1. The principal block

\begin{tabular}{rrrrrrrrrr}
\hline & $\Phi_{1}$ & $\Phi_{2}$ & $\Phi_{3}$ & $\Phi_{4}$ & $\Phi_{5}$ & $\Phi_{6}$ & $\Phi_{7}$ & $\Phi_{8}$ & $\Phi_{9}$ \\
\hline 1 & 1 &. &. &. &. &. &. &. &. \\
406 &. & 1 &. &. &. &. &. &. &. \\
21750 &. &. & 1 & 1 &. &. &. &. &. \\
34944 &. &. &. &. & 1 &. &. &. &. \\
34944 &. &. &. &. & 1 &. &. &. &. \\
45500 &. &. &. &. &. & 1 &. &. &. \\
52780 &. &. & 1 & 1 &. &. & 1 &. &. \\
63336 &. & 1 &. &. &. & 1 &. & 1 &. \\
65975 & 1 &. &. &. & 1 &. & 1 &. &. \\
75400 &. &. & 1 &. &. &. & 1 &. &. \\
76125 & 1 &. &. &. &. & 1 & 1 &. &. \\
102400 &. &. &. & 1 &. & 1 & 1 & 1 &. \\
105560 &. &. & 1 & 1 & 1 &. & 1 & 1 &. \\
118784 &. &. & 1 & 1 & 1 &. & 1 &. & 1 \\
\hline
\end{tabular}

Received by the editor July $21,1992$.

1991 Mathematics Subject Classification. Primary 20-04, 20C20, 20C34, 20C40, 20 D08.

This paper is a contribution to the DFG research project "Algorithmic Number Theory and Algebra". 


\begin{tabular}{crc}
\hline Char. & Degree & \multicolumn{1}{c}{ Factors } \\
\hline$\phi_{1}$ & 1 & 1 \\
$\phi_{2}$ & 406 & $(2)(7)(29)$ \\
$\phi_{3}$ & 13310 & $(2)(5)(11)^{3}$ \\
$\phi_{4}$ & 8440 & $(2)^{3}(5)(211)$ \\
$\phi_{5}$ & 34944 & $(2)^{7}(3)(7)(13)$ \\
$\phi_{6}$ & 45094 & $(2)(7)(3221)$ \\
$\phi_{7}$ & 31030 & $(2)(5)(29)(107)$ \\
$\phi_{8}$ & 17836 & $(2)^{2}(7)^{3}(13)$ \\
$\phi_{9}$ & 31060 & $(2)^{2}(5)(1553)$ \\
\hline
\end{tabular}

1.2. Block 2. This is a block of defect 1 containing characters of the simple group.

\begin{tabular}{rrr}
\hline & $\Phi_{1}$ & $\Phi_{2}$ \\
\hline 3276 & 1 & \\
20475 & $\dot{1}$ & 1 \\
23751 & 1 & 1 \\
\hline
\end{tabular}

\begin{tabular}{crc}
\hline Char. & Degree & Factors \\
\hline$\phi_{1}$ & 3276 & $(2)^{2}(3)^{2}(7)(13)$ \\
$\phi_{2}$ & 20475 & $(3)^{2}(5)^{2}(7)(13)$ \\
\hline
\end{tabular}

1.3. Block 3. This is a block of defect 1 containing characters of the simple group.

\begin{tabular}{rrr}
\hline & $\Phi_{1}$ & $\Phi_{2}$ \\
\hline 3654 & 1 &. \\
91350 & $\dot{1}$ & 1 \\
95004 & 1 & 1 \\
\hline
\end{tabular}

\begin{tabular}{crc}
\hline Char. & Degree & Factors \\
\hline$\phi_{1}$ & 3654 & $(2)(3)^{2}(7)(29)$ \\
$\phi_{2}$ & 91350 & $(2)(3)^{2}(5)^{2}(7)(29)$ \\
\hline
\end{tabular}

1.4. Block 4. This is the nonprincipal block of maximal defect.

\begin{tabular}{|c|c|c|c|c|c|c|c|c|c|}
\hline & $\Phi_{1}$ & $\Phi_{2}$ & $\Phi_{3}$ & $\Phi_{4}$ & $\Phi_{5}$ & $\Phi_{6}$ & $\Phi_{7}$ & $\Phi_{8}$ & $\Phi_{\mathrm{S}}$ \\
\hline 28 & 1 & & & . & . & . & . & 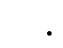 & \\
\hline 28 & . & 1 & & . & . & . & . & . & \\
\hline 1248 & ${ }^{\circ}$ & . & 1 & . & . & ${ }^{\circ}$ & • & . & \\
\hline 1248 & . & . & 1 & & . & • & . & & \\
\hline 7280 & . & . & 1 & 1 & & . & . & . & \\
\hline 7280 & . & . & 1 & . & 1 & & . & . & \\
\hline 8192 & . & . & 1 & . & . & 1 & & & \\
\hline 8192 & & . & 1 & . & . & . & 1 & & \\
\hline 10556 & 1 & & . & . & . & . & . & 1 & \\
\hline 10556 & . & 1 & • & . & . & . & . & & 1 \\
\hline 34944 & & ${ }^{\circ}$ & & & & 1 & 1 & 1 & 1 \\
\hline 38976 & & . & 2 & 1 & 1 & 1 & 1 & 1 & \\
\hline 38976 & ( & . & 2 & 1 & 1 & 1 & 1 & & 1 \\
\hline 48256 & . & 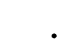 & 1 & 1 & 1 & 1 & 1 & 1 & 1 \\
\hline
\end{tabular}




\begin{tabular}{crc}
\hline Char. & Degree & \multicolumn{1}{c}{ Factors } \\
\hline$\phi_{1}$ & 28 & $(2)^{2}(7)$ \\
$\phi_{2}$ & 28 & $(2)^{2}(7)$ \\
$\phi_{3}$ & 1248 & $(2)^{5}(3)(13)$ \\
$\phi_{4}$ & 6032 & $(2)^{4}(13)(29)$ \\
$\phi_{5}$ & 6032 & $(2)^{4}(13)(29)$ \\
$\phi_{6}$ & 6944 & $(2)^{5}(7)(31)$ \\
$\phi_{7}$ & 6944 & $(2)^{5}(7)(31)$ \\
$\phi_{8}$ & 10528 & $(2)^{5}(7)(47)$ \\
$\phi_{9}$ & 10528 & $(2)^{5}(7)(47)$ \\
\hline
\end{tabular}

1.5. Block 5. This is a block of defect 1 containing faithful characters.

\begin{tabular}{rrr}
\hline & $\Phi_{1}$ & $\Phi_{2}$ \\
\hline 3276 & 1 &. \\
4032 & & 1 \\
7308 & 1 & 1 \\
\hline
\end{tabular}

\begin{tabular}{ccc}
\hline Char. Degree & Factors \\
\hline$\phi_{1}$ & 3276 & $(2)^{2}(3)^{2}(7)(13)$ \\
$\phi_{2}$ & 4032 & $(2)^{6}(3)^{2}(7)$ \\
\hline
\end{tabular}

1.6. Block 6. This is the dual of Block 5 , so the tables are the same.

\section{Proofs}

The distribution of the characters into blocks is easily determined with the help of the character table. It is also fairly easy to find the number of irreducible Brauer characters in each block. The two blocks of maximal defect each contain 14 ordinary and 9 modular irreducible characters. The blocks containing only three ordinary irreducible characters are blocks of defect 1 . Their decomposition matrix is therefore trivially determined.

In the following proofs, PIM stands for projective indecomposable module. We have implicitly assumed that a splitting 3-modular system for $2 \mathrm{Ru}$ is given. Ordinary and Brauer characters are sometimes denoted by their degrees.

2.1. Proof for Block 4. We start with the proof for the nonprincipal block of maximal defect (Block 4) since it is easier than the one for the principal block and since we are going to use some of these results for the proof of the principal block.

We first consider the set of projective characters displayed in Table 1 (next page). Here, $\boldsymbol{\Phi}=\chi_{15}+\chi_{16}+\chi_{23}+\chi_{33}+\chi_{36}$ is the projective character obtained by inducing the character of degree 3 of the maximal subgroup $\left(2^{2} \times \mathrm{Sz}(8)\right): 3$ to the Rudvalis group and restricting it to the principal block.

These projectives show that the decomposition matrix has wedge shape. Therefore, all but $\Lambda_{3}$ are indecomposable. The irreducible Brauer character of degree 1248 is real-valued. Since it is no constituent of $10556_{1}$, it cannot be one of $10556_{2}$ either, the complex conjugate of $10556_{1}$. Therefore, $\Phi_{3}:=\Lambda_{3}-\Lambda_{9}$ is a projective character. It is clear that it is indecomposable. This completes the proof for Block 4 . 
TABLE 1. Some projectives for Block 4

\begin{tabular}{rccccccccc}
\hline & $\chi_{38}$ & $\chi_{41}$ & $\chi_{38}$ & $\chi_{46}$ & $\chi_{45}$ & $\chi_{49}$ & $\chi_{50}$ & $\chi_{44}$ & $\chi_{43}$ \\
& $\otimes$ & $\otimes$ & $\otimes$ & $\otimes$ & $\otimes$ & $\otimes$ & $\otimes$ & $\otimes$ & $\otimes$ \\
& $\chi_{2}$ & $\chi_{2}$ & $\Phi$ & $\chi_{2}$ & $\chi_{2}$ & $\chi_{2}$ & $\chi_{2}$ & $\chi_{2}$ & $\chi_{2}$ \\
\hline & $\Lambda_{1}$ & $\Lambda_{2}$ & $\Lambda_{3}$ & $\Lambda_{4}$ & $\Lambda_{5}$ & $\Lambda_{6}$ & $\Lambda_{7}$ & $\Lambda_{8}$ & $\Lambda_{9}$ \\
\hline 28 & 1 &. &. &. &. &. &. &. &. \\
28 &. & 1 &. &. &. &. &. &. &. \\
1248 &. &. & 1 &. &. &. &. &. &. \\
1248 &. &. & 1 &. &. &. &. &. &. \\
7280 &. &. & 1 & 1 &. &. &. &. &. \\
7280 &. &. & 1 &. & 1 &. &. &. &. \\
8192 &. &. & 1 &. &. & 1 &. &. &. \\
8192 &. &. & 1 &. &. &. & 1 &. &. \\
10556 & 1 &. &. &. &. &. &. & 1 &. \\
10556 &. & 1 & 1 &. &. &. &. &. & 1 \\
34944 &. &. & 1 &. &. & 1 & 1 & 1 & 1 \\
38976 &. &. & 2 & 1 & 1 & 1 & 1 & 1 &. \\
38976 &. &. & 3 & 1 & 1 & 1 & 1 &. & 1 \\
48256 &. &. & 2 & 1 & 1 & 1 & 1 & 1 & 1 \\
\hline
\end{tabular}

2.2. Proof for the principal block. We begin with the set of projective characters displayed in Table 2. The origin of these characters is documented below.

\begin{tabular}{cc}
\hline Char. & Origin \\
\hline$\Lambda_{1}:$ & $\chi_{3} \otimes \chi_{2}$ \\
$\Lambda_{2}:$ & $\chi_{5} \otimes \chi_{2}$ \\
$\Lambda_{3}:$ & $\chi_{37} \otimes \Phi_{7,4}$ \\
$\Lambda_{4}:$ & $\chi_{37} \otimes \chi_{58}$ \\
$\Lambda_{5}:$ & $\operatorname{Ind}_{M_{1}}\left(\theta_{24}\right)$ \\
$\Lambda_{6}:$ & $\operatorname{Ind}_{M_{1}}\left(\theta_{15}\right)$ \\
$\Lambda_{7}:$ & $\operatorname{Ind}_{M_{3}}\left(\theta_{4}\right)$ \\
$\Lambda_{8}:$ & $\chi_{6} \otimes \chi_{2}$ \\
$\Lambda_{9}:$ & $\chi_{7} \otimes \chi_{2}$ \\
$\Lambda_{10}:$ & $\operatorname{Ind}_{M_{1}}\left(\theta_{14}\right)$ \\
\hline
\end{tabular}

In this table the following notations are used. $M_{i}$ denotes the $i$ th maximal subgroup of the Rudvalis group, where the subgroups are sorted in decreasing order. We shall only need to induce characters for the proof of the principal block, and so we only need to consider the maximal subgroups of the Rudvalis group rather than its covering group. Only the three largest maximal subgroups will be used. In ATLAS [2] notation these are: $M_{1}={ }^{2} F_{4}(2), M_{2}=\left(2^{6}: U_{3}(3)\right): 2$ and $M_{3}=\left(2^{2} \times \mathrm{Sz}(8)\right): 3$. The character tables of these maximal subgroups are available in the CAS system (see [3]). The numbering of the characters of 
TABLE 2. Some projectives for the principal block

\begin{tabular}{|c|c|c|c|c|c|c|c|c|c|c|}
\hline & $\Lambda_{1}$ & $\Lambda_{2}$ & $\Lambda_{3}$ & $\Lambda_{4}$ & $\Lambda_{5}$ & $\Lambda_{6}$ & $\Lambda_{7}$ & $\Lambda_{8}$ & $\Lambda_{9}$ & $\Lambda_{10}$ \\
\hline 1 & 1 & & . & . & & . & . & . & . & \\
\hline 406 & . & 1 & • & & $\cdot$ & . & $\cdot$ & $\cdot$ & . & 1 \\
\hline 21750 & 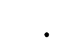 & & 1 & 1 & . & . & & . & . & \\
\hline 34944 & $\cdot$ & $\cdot$ & 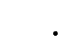 & 1 & 1 & 1 & 1 & 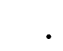 & 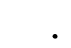 & 1 \\
\hline 34944 & . & & . & 1 & 1 & 1 & 1 & & . & 1 \\
\hline 45500 & . & 1 & 1 & 1 & 1 & & 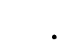 & 1 &  & 2 \\
\hline 52780 & . & & 2 & 1 & 1 & . & . & & 1 & \\
\hline 63336 & & 1 & 2 & 2 & 1 & 1 & 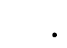 & 1 & . & 2 \\
\hline 65975 & 1 & & 1 & 1 & 2 & 1 & 1 & & 1 & 1 \\
\hline 75400 & & & 4 & 1 & 1 & & . & 1 & 1 & \\
\hline 76125 & 1 & & 2 & 1 & 2 & 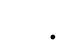 & . & 1 & 1 & 1 \\
\hline 102400 & . & & 4 & 2 & 2 & 1 & . & 1 & 1 & 1 \\
\hline 105560 & . & & 3 & 3 & 2 & 2 & 1 & & 1 & 1 \\
\hline 118784 & & & 5 & 2 & 2 & 1 & 1 & 1 & 1 & 1 \\
\hline
\end{tabular}

the maximal subgroups follows the numbering in the CAS tables. Character $\theta_{24}$ of $M_{1}$ is of degree $675, \theta_{15}$ and $\theta_{14}$ have degree 351 and character $\theta_{4}$ of $M_{3}$ has degree 3 . All these characters are therefore of defect 0 , and hence induce to projective characters.

The character named $\Phi_{7,4}$ is the projective indecomposable character $\Phi_{7}$ of Block 4. A similar notation will be used later on.

Of course, the above induced characters and tensor products may have some constituents outside the principal block. The complete decomposition of these characters is given in the appendix.

Now observe that $\chi_{37} \otimes \chi_{15}=\chi_{40}+\chi_{57}+\chi_{58}+\chi_{59}+2 \chi_{60}+\chi_{61}$, and that all characters but $\chi_{40}$ occurring in this tensor product are of defect 0 . If we denote the characters by their degrees, we can write

$$
28 \otimes 34944=1248+\text { defect } 0 \text { characters }
$$

Since 1248 is irreducible as Brauer character, it follows that 34944 also is an irreducible Brauer character.

We claim that $\left\{\Lambda_{1}, \ldots, \Lambda_{9}\right\}$ is a basic set of projective characters. That is to say, every projective indecomposable character of the principal block is a $\mathbb{Z}$-linear combination of $\left\{\Lambda_{1}, \ldots, \Lambda_{9}\right\}$. This follows from the fact that the matrix of scalar products of $\left\{\Lambda_{1}, \ldots, \Lambda_{9}\right\}$ with the ordinary characters of degrees $1,406,21750,34944_{1}, 45500,52780,63336,75400$, and 102400 is unimodular, which can easily be checked from Table 2 .

Since $\Lambda_{7}$ has inner product 1 with 34944 and inner product 0 with all the other characters listed above, it follows that $\Lambda_{7}$ is the projective indecomposable corresponding to the irreducible 34944 . 
TABLE 3. New basic set of projectives for the principal block

\begin{tabular}{|c|c|c|c|c|c|c|c|c|c|}
\hline & $\Lambda_{1}$ & $\Lambda_{2}$ & $\Lambda_{3}$ & $\Lambda_{11}$ & $\Lambda_{7}$ & $\Lambda_{8}$ & $\Lambda_{12}$ & $\Lambda_{9}$ & $\Lambda_{13}$ \\
\hline 1 & 1 & & & & & . & & . & \\
\hline 406 & $\cdot$ & 1 & & & & $\cdot$ & & & \\
\hline 21750 & . & 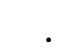 & 1 & 1 & . & . & . & . & \\
\hline 34944 & . & $\cdot$ & . & . & 1 & . & . & . & \\
\hline 34944 & . & & • & 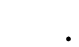 & 1 & . & . & . & \\
\hline 45500 & . & 1 & 1 & 1 & . & 1 & 1 & & \\
\hline 52780 & . & 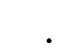 & 2 & 1 & 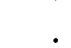 & . & 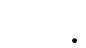 & 1 & \\
\hline 63336 & & 1 & 2 & 2 & & 1 & 1 & & 1 \\
\hline 65975 & 1 & $\cdot$ & 1 & & 1 & . & • & 1 & \\
\hline 75400 & & & 4 & 1 & 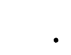 & 1 & & 1 & \\
\hline 76125 & 1 & . & 2 & 1 & 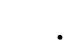 & 1 & 1 & 1 & \\
\hline 102400 & 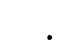 & . & 4 & 2 & & 1 & 1 & 1 & 1 \\
\hline 105560 & . & . & 3 & 2 & 1 & . & . & 1 & 1 \\
\hline 118784 & & & 5 & 1 & 1 & 1 & 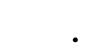 & 1 & \\
\hline
\end{tabular}

It is clear the $\Lambda_{1}$ and $\Lambda_{2}$ are PIM's (since they have only 3 ordinary constituents). Also, 406 is irreducible, since it has inner product 1 with $\Lambda_{2}$ and inner product 0 with each of $\left\{\Lambda_{3}, \ldots, \Lambda_{9}\right\}$ and $\Lambda_{1}$.

Each of $\Lambda_{2}$ and $\Lambda_{7}$ can therefore be subtracted from any other projective as many times as 406 respectively 34944 is contained in that projective. If we do so, we obtain the new basic set of projectives displayed in Table 3.

The new projectives were obtained as follows.

\begin{tabular}{ll}
\hline Char. & \multicolumn{1}{c}{ Origin } \\
\hline$\Lambda_{11}:$ & $\Lambda_{4}-\Lambda_{7}$ \\
$\Lambda_{12}:$ & $\Lambda_{10}-\Lambda_{2}-\Lambda_{7}$ \\
$\Lambda_{13}:$ & $\Lambda_{6}-\Lambda_{7}$
\end{tabular}

It is easy to check (consider the character degrees modulo 9), that of the new projectives $\Lambda_{12}$ and $\Lambda_{13}$ are indecomposable. To proceed, we consider some more projectives which are displayed in Table 4 . The origin of these characters is documented in the following table.

\begin{tabular}{cc}
\hline Char. & Origin \\
\hline$\Lambda_{14}:$ & $\operatorname{Ind}_{M_{2}}\left(\theta_{13}\right)$ \\
$\Lambda_{15}:$ & $\operatorname{Ind}_{M_{1}}\left(\theta_{23}\right)$ \\
$\Lambda_{16}:$ & $\chi_{37} \otimes \Phi_{5,4}$ \\
$\Lambda_{17}:$ & $\operatorname{Ind}_{M_{2}}\left(\theta_{12}\right)$ \\
$\Lambda_{18}:$ & $\chi_{37} \otimes \Phi_{3,4}$ \\
\hline
\end{tabular}


TABLE 4. More projectives for the principal block

\begin{tabular}{rrrrrr}
\hline & $\Lambda_{14}$ & $\Lambda_{15}$ & $\Lambda_{16}$ & $\Lambda_{17}$ & $\Lambda_{18}$ \\
\hline 1 &. &. &. &. &. \\
406 &. &. &. &. &. \\
21750 &. & 1 & 1 & 1 & 2 \\
34944 & 1 & 1 &. & 3 & 1 \\
34944 & 1 & 1 &. & 3 & 1 \\
45500 & 1 & 2 &. & 3 &. \\
52780 & 1 & 1 & 2 & 1 & 3 \\
63336 & 3 & 2 & 2 & 3 & 3 \\
65975 & 2 & 1 & 1 & 3 & 2 \\
75400 & 3 & 1 & 3 &. & 5 \\
76125 & 2 & 2 & 1 & 3 & 1 \\
102400 & 4 & 2 & 3 & 4 & 5 \\
105560 & 4 & 2 & 4 & 4 & 7 \\
118784 & 4 & 2 & 3 & 4 & 7 \\
\hline
\end{tabular}

The characters $\theta_{13}$ and $\theta_{12}$ of $M_{2}$ are of degree 27 and $\theta_{23}$ of $M_{1}$ is of degree 675 , thus all three are of defect 0 . The projectives, denoted $\Phi_{5,4}$ and $\Phi_{3,4}$, are the projective indecomposables $\Phi_{5}$, respectively $\Phi_{3}$, of Block 4 .

TABLE 5. Projectives of Table 4 improved

\begin{tabular}{rrrrrr}
\hline & $\Lambda_{19}$ & $\Lambda_{20}$ & $\Lambda_{21}$ & $\Lambda_{22}$ & $\Lambda_{23}$ \\
\hline 1 &. &. &. &. &. \\
406 &. &. &. &. &. \\
21750 &. & 1 & 1 & 1 & 2 \\
34944 &. &. &. &. &. \\
34944 &. &. &. &. &. \\
45500 & 1 & 2 &. & 3 &. \\
52780 & 1 & 1 & 2 & 1 & 3 \\
63336 & 3 & 2 & 2 & 3 & 3 \\
65975 & 1 &. & 1 &. & 1 \\
75400 & 3 & 1 & 3 &. & 5 \\
76125 & 2 & 2 & 1 & 3 & 1 \\
102400 & 4 & 2 & 3 & 4 & 5 \\
105560 & 3 & 1 & 4 & 1 & 6 \\
118784 & 3 & 1 & 3 & 1 & 6 \\
\hline
\end{tabular}


TABLE 6. New basic set of projectives for the principal block

\begin{tabular}{rrrrrrrrrr}
\hline & $\Lambda_{1}$ & $\Lambda_{2}$ & $\Lambda_{24}$ & $\Lambda_{25}$ & $\Lambda_{7}$ & $\Lambda_{12}$ & $\Lambda_{9}$ & $\Lambda_{13}$ & $\Lambda_{26}$ \\
\hline 1 & 1 &. &. &. &. &. &. &. &. \\
406 &. & 1 &. &. &. &. &. &. &. \\
21750 &. &. & 1 & 1 &. &. &. &. &. \\
34944 &. &. &. &. & 1 &. &. &. &. \\
34944 &. &. &. &. & 1 &. &. &. &. \\
45500 &. &. &. &. &. & 1 &. &. &. \\
52780 &. &. & 2 & 1 &. &. & 1 &. &. \\
63336 &. &. &. &. &. & 1 &. & 1 &. \\
65975 & 1 &. & 1 &. & 1 &. & 1 &. &. \\
75400 &. &. & 4 & 1 &. &. & 1 &. & 1 \\
76125 & 1 &. & 1 &. &. & 1 & 1 &. &. \\
102400 &. &. & 2 &. &. & 1 & 1 & 1 &. \\
105560 &. &. & 2 & 1 & 1 &. & 1 & 1 &. \\
118784 &. &. & 5 & 1 & 1 &. & 1 &. & 1 \\
\hline
\end{tabular}

We again subtract off $\Lambda_{7}$ as often as possible and obtain the projectives of Table 5. In terms of the basic set of Table 3 , the characters can be written as follows.

\begin{tabular}{rrrrrrrrrr}
\hline & $\Lambda_{1}$ & $\Lambda_{2}$ & $\Lambda_{3}$ & $\Lambda_{11}$ & $\Lambda_{7}$ & $\Lambda_{8}$ & $\Lambda_{12}$ & $\Lambda_{9}$ & $\Lambda_{13}$ \\
\hline$\Lambda_{19}$ &. &. &. &. &. & 2 & -1 & 1 & 2 \\
$\Lambda_{20}$ &. &. &. & 1 &. &. & 1 &. & -1 \\
$\Lambda_{21}$ &. &. &. & 1 &. & 1 & -2 & 1 & 1 \\
$\Lambda_{22}$ &. &. & 1 &. &. & -3 & 5 & -1 & -1 \\
$\Lambda_{23}$ &. &. & 1 & 1 &. &. & -2 &. & 1
\end{tabular}

This gives us the new basic set of projectives of Table 6 . The new projectives were obtained as follows.

\begin{tabular}{ll}
\hline Char. & \multicolumn{1}{c}{ Origin } \\
\hline$\Lambda_{24}:$ & $\Lambda_{3}-\Lambda_{12}-\Lambda_{13}$ \\
$\Lambda_{25}:$ & $\Lambda_{11}-\Lambda_{12}-\Lambda_{13}$ \\
$\Lambda_{26}:$ & $\Lambda_{8}-\Lambda_{12}$
\end{tabular}

That these characters are indeed projective can easily be deduced from the projectives of Table 5. Consider, for example, the projective $\Lambda_{19}$. Its expression in terms of the basic set implies $\Lambda_{19}+\Lambda_{12}=2 \Lambda_{8}+\Lambda_{9}+2 \Lambda_{13}$. Since $\Lambda_{12}$ is indecomposable, this implies that $\Lambda_{12}$ is contained in $\Lambda_{8}$. Similarly, $\Lambda_{20}$ shows that $\Lambda_{13}$ is contained in $\Lambda_{11}$.

Of the new projective characters, $\Lambda_{26}$ is indecomposable. Now $\Lambda_{24}=\Lambda_{22}$ $3 \Lambda_{12}+\Lambda_{9}+3 \Lambda_{26}$. This implies that $\Lambda_{27}:=\Lambda_{22}-3 \Lambda_{12}$ is a projective character. 
TABLE 7. Hypothetical decomposition matrix

\begin{tabular}{|c|c|c|c|c|c|c|c|c|c|}
\hline & $\Phi_{1}^{\prime}$ & $\Phi_{2}^{\prime}$ & $\Phi_{3}^{\prime}$ & $\Phi_{4}^{\prime}$ & $\Phi_{5}^{\prime}$ & $\Phi_{6}^{\prime}$ & $\Phi_{7}^{\prime}$ & $\Phi_{8}^{\prime}$ & $\boldsymbol{\Phi}_{9}^{\prime}$ \\
\hline 1 & 1 & & & & & & & & \\
\hline 406 & . & 1 & & & & & & & \\
\hline 21750 & . & . & 1 & & & & . & & \\
\hline 3494 & • & • & . & 1 & & & . & & \\
\hline 34944 & . & & . & 1 & & & & & \\
\hline 45 & . & 1 & & . & 1 & & & & \\
\hline $52^{\prime}$ & . & . & 1 & & & 1 & & & \\
\hline 63336 & & 1 & . & & 1 & & 1 & & \\
\hline 65975 & 1 & . & . & 1 & . & 1 & . & & \\
\hline 75 & & & . & • & & & & 1 & \\
\hline 7 & 1 & ${ }^{\circ}$ & . & . & 1 & 1 & & & \\
\hline 102400 & . & . & & . & 1 & 1 & 1 & & 1 \\
\hline 105560 & & & 1 & 1 & . & 1 & 1 & & \\
\hline 118784 & & & . & 1 & • & . & . & 1 & 1 \\
\hline
\end{tabular}

Substituting $\Lambda_{24}$ by $\Lambda_{27}$, and renaming the characters, we obtain the matrix displayed in $\S 1.1$. We proceed to show that this matrix already is the matrix of decomposition numbers. To do so, we only need to show that $\Phi_{3}, \Phi_{4}$ and $\Phi_{7}$ are characters of indecomposable modules.

For this purpose we consider the maximal subgroup $M_{2}=\left(2^{6}: U_{3}(3)\right): 2$ of the Rudvalis group. Let $\overline{1}$ denote the linear character (and the corresponding module) of $M_{2}$ which takes value -1 outside $2^{6}: U_{3}(3)$. We obtain

$$
\operatorname{Ind}_{M_{2}}(\overline{1})=\chi_{9}+\chi_{25}+\chi_{28}
$$

In terms of degrees, this is

$$
\operatorname{Ind}_{M_{2}}(\overline{1})=21750+75400+91350 .
$$

Let $X$ denote the component in the principal block of the corresponding induced module. Then the Brauer character of $X$ is $21750+75400$. Since $\overline{1}$ is a trivial source module, so is $X$.

Now suppose for the moment that $X$ is not indecomposable. Then it decomposes as $X=X_{1} \oplus X_{2}$, where $X_{1}$ has Brauer character 21750, say, and $X_{2}$ has Brauer character 75400 . Each of $X_{1}$ and $X_{2}$ has trivial source, and so a 1-dimensional endomorphism ring. Since all characters in the principal block are real-valued, this implies that $X_{1}$ and $X_{2}$ are simple.

Now $\Phi_{9}$ is indecomposable with inner product 1 with the irreducible 75400 , so $\Phi_{9}$ is the character of the projective cover of 75400 . Thus, $\Phi_{3}-\Phi_{9}$ and $\Phi_{7}-\Phi_{9}$ are projective indecomposable characters. Moreover, $\Phi_{3}-\Phi_{9}$ has inner product 1 with 21750 and can therefore be subtracted from $\Phi_{4}$. This gives all the projective indecomposable characters and therefore the hypothetical 
decomposition matrix displayed in Table 7. The second table below gives the degrees of the irreducible Brauer characters.

\begin{tabular}{cr}
\hline Char. Degree \\
\hline$\phi_{1}^{\prime}$ & 1 \\
$\phi_{2}^{\prime}$ & 406 \\
$\phi_{3}^{\prime}$ & 21750 \\
$\phi_{4}^{\prime}$ & 34944 \\
$\phi_{5}^{\prime}$ & 45094 \\
$\phi_{6}^{\prime}$ & 31030 \\
$\phi_{7}^{\prime}$ & 17836 \\
$\phi_{8}^{\prime}$ & 75400 \\
$\phi_{9}^{\prime}$ & 8440 \\
\hline
\end{tabular}

Consider $\Phi_{9}^{\prime}=102400+118748$, the PIM corresponding to the irreducible Brauer character of degree 8440 . As Brauer character, it is $\Phi_{9}^{\prime}=17836+$ $34944+45094+31030+75400+2 \times 8440$. Since every simple module in the principal block is self-dual, this implies that the radical modulo the socle of the projective indecomposable module corresponding to $\Phi_{9}^{\prime}$ is semisimple. It therefore has five direct summands, contradicting a result of Webb (see [1, 2.31.5]).

Thus $X$ is indecomposable. Now $X$ is self-dual and has a 2-dimensional endomorphism ring. Again, since every irreducible module in the block is selfdual, it follows that the head and the socle of $X$ are simple and isomorphic. By the projective characters we now know that neither 21750 nor 75400 has repeated composition factors. Therefore, 21750 and 75400 have a common composition factor. This implies that there is a PIM containing 21750 and 75400 , and so $\Phi_{3}$ is indecomposable (since there is just one possible way $\Phi_{3}$ can split into a sum of two projectives).

Suppose now that $\Phi_{4}=\Phi_{4}^{\prime}+\Phi_{4}^{\prime \prime}$ is the sum of two projectives. Then, as is easily checked by looking at the character degrees modulo 9 , each of $\Phi_{4}^{\prime}$ and $\Phi_{4}^{\prime \prime}$ is a PIM and we may assume that $\Phi_{4}^{\prime \prime}=102400+118748$. But then $\Phi_{3}=\Phi_{4}^{\prime}+\Phi_{9}$, which is absurd since the projective indecomposable characters are linearly independent. Thus $\Phi_{4}$ is indecomposable.

We have now found 8 out of 9 PIM's. Hence, either $\Phi_{7}$ is indecomposable or it contains $\Phi_{9}$ as a component. This is equivalent to saying that 75400 either has 3 or 2 composition factors.

To decide this question, we again consider the maximal subgroup $M_{3}=$ $\left(2^{2} \times \mathrm{Sz}(8)\right): 3$. The principal block consists of the three 1-dimensional characters $1, \omega$ and $\bar{\omega}$ corresponding to the factor group of order 3 . In the principal block of $2 \mathrm{Ru}$ we have

$$
\operatorname{Ind}_{M_{3}}(1)=\chi_{1}+\chi_{15}+\chi_{16}+\chi_{21}+\chi_{23}+\chi_{25}+\chi_{32}
$$

and

$$
\operatorname{Ind}_{M_{3}}(\omega)=\operatorname{Ind}_{M_{3}}(\bar{\omega})=\chi_{23}+\chi_{26}+\chi_{33}+\chi_{36}
$$


This shows that the restriction of $\chi_{9}=21750$ to $M_{3}$ has no component in the principal block. Then the same is true for each modular constituent of 21750 .

Suppose for a contradiction that $\chi_{25}=75400$ has just 2 modular constituents. Let $\phi$ be that one which is not in 21750. Then the restriction of $\phi$ to the principal block of $M_{3}$ is the trivial character.

Then, by Frobenius reciprocity, $\phi$ is contained in the head and in the socle of $\operatorname{Ind}_{M_{3}}(1)$. On the other hand, by the decomposition numbers we now know that the multiplicity of $\phi$ as a composition factor of $\operatorname{Ind}_{M_{3}}(1)$ is just 1 . This shows that $\phi$ is a direct summand of this permutation module. But then $\phi$ is liftable to an ordinary character, as is well known. This contradiction shows that 75400 has 3 composition factors and hence completes the proof.

\section{APPENDIX. INDUCED CHARACTERS AND TENSOR PRODUCTS}

The first table gives the decomposition of some induced characters and some tensor products.

\begin{tabular}{|c|c|c|c|c|c|c|c|c|c|c|c|c|c|c|c|c|c|c|c|}
\hline$\theta:$ & 1 & 2 & 3 & 4 & 5 & 6 & 7 & 8 & 9 & & 1 & 12 & 13 & 14 & 15 & 16 & & & 9 \\
\hline 1 & & $\cdot$ & $\cdot$ & . & $\cdot$ & 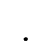 & . & 1 & & & & 1 & & & & & & & \\
\hline 378 & & & . & . & & . & ${ }^{\circ}$ & • & . & & . & & 1 & & & & & & \\
\hline 3 & & . &. & . & . & 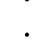 & & . & & & & & & & & & & & \\
\hline 40 & 1 & & 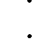 & ${ }^{\circ}$ & . & & 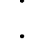 & ${ }^{\circ}$ & . & & & & 1 & & & & & & \\
\hline 7 & & & & . & $\cdot$ & $\dot{1}$ & & & . & & $i$ & $i$ & & & & & & & \\
\hline 3276 & 1 & 1 & 1 & & 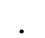 & 2 & . & 1 & . & . & & . & & & & & & & \\
\hline 3 & 1 & 2 & 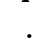 & & . & 1 & & & . & & & & & & 1 & & & . & \\
\hline 204 & & . & $\cdot$ & . & . &  & 1 & 1 & . & . & & . & & & 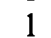 & & & & \\
\hline & & . & 1 & . & 1 & 1 & . & . & . & & . & $\cdot$ & $\cdot$ & 1 & 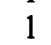 & & $\cdot$ & $i$ & $i$ \\
\hline & 1 & 1 & 1 & & 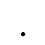 & 2 & 1 & 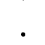 & 1 & 1 & & & & & 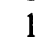 & & & . & \\
\hline & 1 & 1 & 1 & 1 & . & 3 & 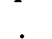 & . & 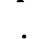 & & 1 & & & & 1 & & & & \\
\hline & 1 & 1 & 1 & 1 & . & 3 & . & 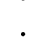 & . & & 1 & & & & 1 & & & & \\
\hline & 1 & 1 & 1 & 1 & 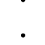 & 3 & & 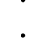 & & & 1 & & & . & 1 & & & & \\
\hline & 1 & 2 & 1 & 1 & . & 4 & 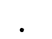 & 1 & 1 & 1 & 1 & & . & . & & & 1 & . & \\
\hline & 1 & 1 & 1 & 1 & . & 3 & 1 & 1 & 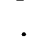 & 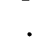 & 1 & & & & $i$ & & . & . & 1 \\
\hline & 1 & 1 & 1 & 1 & . & 3 & 1 & 1 & . & . & 1 & & & & 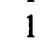 & & & & 1 \\
\hline & 1 & 1 & 1 & 1 & 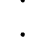 & 2 & 2 & & 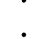 & & 1 & & & & 1 & & & & \\
\hline & 1 & 1 & 1 & 1 & . & 2 & 2 & & . & & 1 & & & & 1 & & • & . & \\
\hline & 1 & 1 & 1 & 1 & & 2 & 2 & 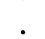 & 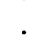 & . & 1 & & & & 1 & & & $\cdot$ & \\
\hline & 2 & 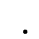 & 2 & 1 & . & 3 & 1 & 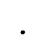 & 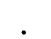 & & 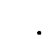 & & 1 & 1 & 1 & 1 & & & \\
\hline & & & 1 & 1 & ${ }^{\circ}$ & 1 & 1 & 1 & . & & & & & & 1 & & 1 & & 3 \\
\hline & 2 & 1 & 2 & 1 & & 3 & 3 & & & & & & 1 & 2 & 2 & 1 & & & 3 \\
\hline & 1 & 1 & 1 & 2 & & 3 & 2 & 1 & 1 & 1 & 1 & 1 & & 1 & 1 & & 1 & & 2 \\
\hline & . & . & 1 & 1 & & 1 & 2 & & . & . & 1 & 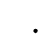 & & 3 & & 1 & 1 & & 3 \\
\hline & & & $i$ & 1 & 1 & & 3 & 1 & & & & & & 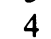 & $i$ & 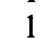 & $*^{*}$ & & 8 \\
\hline & 1 & & 2 & 2 & . & 3 & 2 & 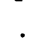 & 1 & 1 & & 1 & & 2 & 2 & 1 & & & 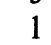 \\
\hline & 1 & 1 & 1 & 2 & & 3 & 3 & & 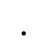 & 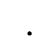 & 2 & & & 3 & 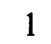 & 1 & & & 3 \\
\hline & & & 2 & 1 & $i$ & 1 & 4 & . & . & & & & 1 & 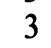 & 1 & & & & 3 \\
\hline & 1 & & 2 & 1 & - & 2 & 4 & & 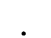 & . & 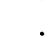 & & 1 & 3 & 2 & 1 & & & 3 \\
\hline & & 1 & 1 & 2 & & 3 & 3 & & 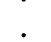 & • & 1 & & . & 3 & 2 & 1 & 1 & & 6 \\
\hline & & 1 & 1 & 2 & $\cdot$ & 3 & 3 & & & • & 1 & & & J & 2 & 1 & 1 & & 6 \\
\hline & 1 & 1 & 2 & 2 & 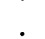 & 4 & 4 & 1 & & & & & & 4 & 2 & 1 & & & 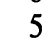 \\
\hline & 1 & 2 & 2 & 2 & 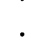 & 4 & 4 & & 1 & 1 & 1 & & & 3 & 3 & & & & 7 \\
\hline & 1 & 1 & 2 & 2 & & 4 & $A$ & & & & 1 & & & 5 & 2 & 1 & & & 6 \\
\hline & 1 & 1 & 2 & 2 & & 4 & 4 & & & & 1 & & & 4 & 2 & . & & & \\
\hline 784 & 1 & 1 & 2 & 2 & & 4 & 4 & & 1 & 1 & 1 & & & $J$ & 2 & 1 & 1 & 3 & 7 \\
\hline
\end{tabular}


The next table gives the tensor products which decompose entirely in faithful characters.

\begin{tabular}{|c|c|c|c|c|c|c|c|c|c|c|}
\hline $\boldsymbol{\theta}:$ & 20 & 21 & 22 & 23 & 24 & 25 & 26 & 27 & 28 & 29 \\
\hline 28 & 1 & & . & . & & . & $\cdot$ & & $\cdot$ & \\
\hline 28 & . & 1 & & & & & 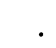 & & . & . \\
\hline 1248 & . & . & 1 & & & & . & & . & \\
\hline 1248 & . & . & 1 & & 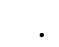 & & 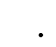 & & . & 1 \\
\hline 3276 & . & . & . & . & . & . & . & . & . & \\
\hline 3276 & . & ${ }^{\circ}$ & . & . & 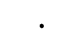 & . & . & 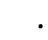 & . & \\
\hline 4032 & . & . & . & . & . & . & . & 1 & & \\
\hline 4032 & . & . & & & &  & . & . & 1 & \\
\hline 7280 & 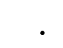 & . & 1 & 1 & & . & 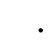 & . & . & \\
\hline 7280 & - & . & 1 & & 1 & . & . & & . & \\
\hline 7308 & & . & • & & & & . & 1 & & \\
\hline 7308 & 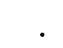 & . & & & . & & . & & 1 & \\
\hline 8192 & & & 1 & & & 1 & & & 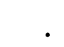 & \\
\hline 8192 & & & 1 & & . & 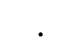 & 1 & & 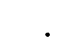 & \\
\hline 10556 & 1 & & & & & & . & 1 & & \\
\hline 10556 & & 1 & 1 & & & & & & 1 & \\
\hline 34944 & & & 1 & & & 1 & 1 & 1 & 1 & \\
\hline 38976 & & & 2 & 1 & 1 & 1 & 1 & 1 & & \\
\hline 38976 & & & 3 & 1 & 1 & 1 & 1 & & 1 & \\
\hline 48256 & & & 2 & 1 & 1 & 1 & 1 & 1 & 1 & \\
\hline 87696 & & 1 & 6 & 1 & 1 & 2 & 2 & 1 & 1 & 1 \\
\hline 98280 & & 1 & 8 & 2 & 2 & 2 & 2 & 1 & & 1 \\
\hline 98280 & & 1 & 9 & 2 & 2 & 2 & 2 & & 1 & 1 \\
\hline 118 & & 2 & 17 & 4 & 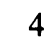 & 5 & 5 & 2 & 2 & 2 \\
\hline 250560 & & 2 & 15 & 5 & 5 & 5 & 5 & 3 & 3 & 1 \\
\hline
\end{tabular}

The following tables document the origin of the projectives, where $\Phi=\chi_{15}+$ $\chi_{16}+\chi_{23}+\chi_{33}+\chi_{36}$ and $\Phi_{i, 4}$ denotes the projective indecomposable sharacter $\Phi_{i}$ of Block 4 .

\begin{tabular}{cc}
\hline Char. & Origin \\
\hline$\Theta_{1}:$ & $\operatorname{Ind}_{M_{1}}\left(\theta_{14}\right)$ \\
$\Theta_{2}:$ & $\operatorname{Ind}_{M_{1}}\left(\theta_{15}\right)$ \\
$\Theta_{3}:$ & $\operatorname{Ind}_{M_{1}}\left(\theta_{23}\right)$ \\
$\Theta_{4}:$ & $\operatorname{Ind}_{M_{1}}\left(\theta_{24}\right)$ \\
$\Theta_{5}:$ & $\operatorname{Ind}_{M_{2}}\left(\theta_{2}\right)$ \\
$\Theta_{6}:$ & $\operatorname{Ind}_{M_{2}}\left(\theta_{12}\right)$ \\
$\Theta_{7}:$ & $\operatorname{Ind}_{M_{2}}\left(\theta_{13}\right)$ \\
$\Theta_{8}:$ & $\operatorname{Ind}_{M_{3}}\left(\theta_{1}\right)$ \\
$\Theta_{9}:$ & $\operatorname{Ind}_{M_{3}}\left(\theta_{2}\right)$ \\
$\Theta_{10}:$ & $\operatorname{Ind}_{M_{3}}\left(\theta_{3}\right)$ \\
$\Theta_{11}:$ & $\operatorname{Ind}_{M_{3}}\left(\theta_{4}\right)$ \\
$\Theta_{12}:$ & $\chi_{3} \otimes \chi_{2}$ \\
$\Theta_{13}:$ & $\chi_{5} \otimes \chi_{2}$ \\
$\Theta_{14}:$ & $\chi_{37} \otimes \Phi_{7,4}$ \\
$\Theta_{15}:$ & $\chi_{37} \otimes \chi_{58}$ \\
\hline
\end{tabular}

\begin{tabular}{cc}
\hline Char. & Origin \\
\hline$\Theta_{16}:$ & $\chi_{6} \otimes \chi_{2}$ \\
$\Theta_{17}:$ & $\chi_{7} \otimes \chi_{2}$ \\
$\Theta_{18}:$ & $\chi_{37} \otimes \Phi_{5,4}$ \\
$\Theta_{19}:$ & $\chi_{37} \otimes \Phi_{3,4}$ \\
$\Theta_{20}:$ & $\chi_{38} \otimes \chi_{2}$ \\
$\Theta_{21}:$ & $\chi_{41} \otimes \chi_{2}$ \\
$\Theta_{22}:$ & $\chi_{38} \otimes \Phi$ \\
$\Theta_{23}:$ & $\chi_{46} \otimes \chi_{2}$ \\
$\Theta_{24}:$ & $\chi_{45} \otimes \chi_{2}$ \\
$\Theta_{25}:$ & $\chi_{49} \otimes \chi_{2}$ \\
$\Theta_{26}:$ & $\chi_{50} \otimes \chi_{2}$ \\
$\Theta_{27}:$ & $\chi_{44} \otimes \chi_{2}$ \\
$\Theta_{28}:$ & $\chi_{43} \otimes \chi_{2}$ \\
$\Theta_{29}:$ & $\chi_{37} \otimes \chi_{15}$ \\
\hline
\end{tabular}




\section{BIBLIOGRAPHY}

1. D. Benson, Modular representation theory: New trends and methods, Lecture Notes in Math., vol. 1081, Springer-Verlag, 1984.

2. J. H. Conway, R. T. Curtis, S. P. Norton, R. A. Parker, and R. A. Wilson, Atlas of finite groups, Oxford Univ. Press, London, 1974.

3. J. Neubüser, H. Pahlings, and W. Plesken, CAS; Design and use of a system for the handling of characters of finite groups, Computational Group Theory, Academic Press, 1984, pp. 195-247.

IWR der Universität Heidelberg, Im Neuenheimer Feld 368, DW-69120 Heidelberg, GERMANY

E-mail address: hiss@euterpe.iwr.uni-heidelberg.de 